\title{
Dietary characteristics of adult women participating in the BiZiFED trial (Biofortified Zinc Flour to Eliminate Zinc Deficiency), Pakistan
}

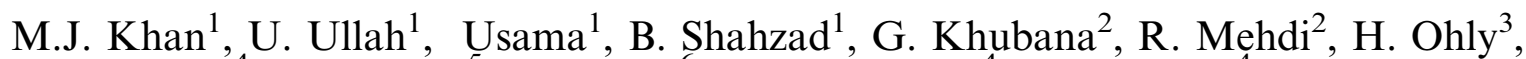 \\ M.R. Broadley ${ }^{4}$, M. Zaman ${ }^{5}$, M.H. Zia ${ }^{6}$, H. J. McArdle ${ }^{4}$, S.D. Young ${ }^{4}$ and N.M. Lowe ${ }^{3}$ \\ ${ }^{1}$ Institute of Basic Medical Sciences, Khyber Medical University Peshawar Pakistan, ${ }^{2}$ Abaseen Foundation Peshawar \\ Pakistan, ${ }^{3}$ Faculty of Health and Wellbeing, University of Central Lancashire Preston PRI $2 \mathrm{HE}$, UK, ${ }^{4}$ School of \\ Biosciences, University of Nottingham, Sutton Bonington Campus Loughborough LE12 5RD, UK, ${ }^{5}$ Lady Reading \\ Hospital Peshawar Pakistan, and ${ }^{6}$ Research and Development Section, Fauji Fertilizer Company Limited, Pakistan.
}

Zinc deficiency is prevalent in low and middle-income countries, including Pakistan where it affects over $40 \%$ of the population ${ }^{(1)}$. BiZiFED is a double blind randomized control crossover trial to test the effectiveness of a new wheat variety (Zincol-2016) in women of child-bearing age in a resource-poor community to address zinc deficiency in Pakistan.

Non-pregnant women $(\mathrm{n}=50)$ were randomly selected from the participating community to consume control flour (Galaxy variety) for a period of 2 weeks. They were then randomized into control (Galaxy 2013 variety; 22.5 mg/kg) and intervention arm (Zincol-2016; $43.2 \mathrm{mg} / \mathrm{kg}$ zinc) for 8 weeks followed by cross over for another 8 weeks. Anthropometric, demographic, and dietary data were collected according to published protocols ${ }^{(2)}$. Dietary data was collected by the study nutritionist using 24 -hour recall and FAO Dietary Diversity questionnaire ${ }^{(3)}$ at baseline (T1), mid (T2) and end (T3) of first intervention period, and mid (T4) and end (T5) of crossover period. Total energy, macro and micronutrient, and dietary fiber intake were calculated from 24-hour dietary recall through Windiet $2005^{\circledR}$ software, while total dietary diversity score (DDS), Women Dietary Diversity Score (WDDS), intake of vitamin A and iron rich foods were calculated from FAO questionnaire according to the FAO guidelines.

No significant difference was observed in demographic and anthropometric characteristics of women in control versus intervention arm at T1. Mean energy intake (kcal) of women at all time points was significantly less than the recommended intake of $2100 \mathrm{kcal}$ (Mean [StDev]; 1609.1(558.4) kcal, p<0.001, 1-sample t-test). There was no significant difference in the energy intake, macro, and micronutrient intake between groups and between treatment arms. Mean Zinc intake was 7.3(0.31) mg/day, significantly lower $(p=0.030)$ than the recommended intake $(8 \mathrm{mg} /$ day $)$. However, this was not different between time points and between groups. The total dietary diversity scores at T1 through to T5 were $11 \cdot 5(2 \cdot 5), 12 \cdot 1(1 \cdot 7), 11 \cdot 6(1 \cdot 4), 11 \cdot 9(1 \cdot 5), 11 \cdot 6(1 \cdot 8)$ respectively $(\mathrm{p}=$ $0 \cdot 517$, one-way ANOVA). The WDDS was in lower range with average of 5 food groups being consumed at individual level (healthy range; $\geqslant 6$ food groups) with no significant difference between the 5 time points and between groups. All participants reported the consumption of cereals and sweets (as per FAO definition). The majority of participants reported the consumption of vitamin A rich vegetables $(94.96 \%)$, white tubers and roots $(89.50 \%)$, dark green leafy vegetables $(98.48 \%)$, and legumes nuts and seeds (93.3\%), and most did not report the use of vitamin A rich fruits (94.96\%), organ meat (79.92\%), and fish (85.36\%) compared to those who did.

Energy intake and dietary micronutrient intake (depicted by low WDDS) were less than the recommended intake in non-pregnant women of child-bearing age which suggests the probability of inadequacy of micronutrients in the diet of these women

This ongoing project is funded by BBSRC. Zincol-NR421 grain was supplied by HarvestPlus, and grown by Fauji Fertilizer Company. Abaseen Foundation facilitated recruitment and sample collection in the field.

1. National Nutrition Survey of Pakistan (2011).

2. Lowe NM et al. (2018) BMJ open (in press)

3. http://www.fao.org/3/a-i1983e.pdf 